\title{
Implementing staff supervision training in a corrections environment
}

Ruth Ford Department of Corrections, New Zealand

\begin{abstract}
INTRODUCTION: Many human service organisations aim to improve the delivery of supervision to their professional staff. In the Aotearoa New Zealand Department of Corrections, changes led to a project which involved the implementation of in-house supervision and supervision training for programme facilitators. This article describes the project and reports on a subsequent review against a retrospective literature review. The content, methods and evaluations of seven deliveries of the week-long supervision training are then critically reflected on through the lens of the literature, with concluding recommendations.

SEARCH STRATEGY AND DATA: The literature review was defined by the use of key terms to search four databases and a library catalogue, resulting in use of 25 articles. While not set up as a formal research project, data from participant evaluations of eight, week-long supervision training courses were analysed, as were results of a national supervision survey.

FINDINGS: Training content, methods and principles were generally well aligned with what is identified as important in the literature, with a few omissions such as assessment processes of supervisors when in the field. Data from participant evaluations showed strong areas of the training (such as skill development through practices) using the model prescribed. A relatively high level of participant satisfaction was demonstrated in the evaluation material. Specific challenges to staff supervision in the Department of Corrections' context were identified with reference to the literature and are discussed.
\end{abstract}

CONCLUSIONS: Seven areas of further focus were identified and recommendations are made with reference to the literature.

KEYWORDS: supervision training; supervision; staff supervision; implementation; corrections
Many human service organisations aim to improve the delivery of supervision to their professional staff. In the New Zealand Department of Corrections (DoC), changes led to a reconfiguring of in-house supervision for programme facilitator staff. The article describes this project and reports on a subsequent review and evaluation of the project. A retrospective literature review was completed with a focus on demonstrating a need for supervision training, the identification of existing barriers to supervision and training, and the perceived benefits. The content, methods and evaluations of eight deliveries of the weeklong supervision training are then critically reflected on through the lens of the literature, with concluding recommendations.

\section{Background}

Programme facilitators are professional staff members in the Department of Corrections who facilitate motivational and rehabilitative programmes with offenders in correctional facilities (prison) and probation service
AOTEAROA NEW ZEALAND SOCIAL WORK 29(3), 95-107.

CORRESPONDENCE TO: Ruth Ford

gaiability@aim.com 
centres (community). The main rehabilitative programmes are based on a variety of psychological and cultural models such as cognitive behavioural therapy, te whare tapa wha (Durie, 1998) and others. Since their appointment in 2000, programme facilitators were provided professional supervision by contracted and departmental psychologists. Over the years, the capacity of programmes staff increased and, in July 2013, the supervision training project began, with a rationale of continuing capacity building within the programme delivery team and to create more time for other aspects of psychologists' work.

In late 2013 there were approximately 166 programme facilitators and managers throughout Aotearoa New Zealand within the specific programme delivery team. The comprehensive organisational policy stipulated professional supervision to be between one and two hours per week for a co-facilitator pair, or an individual, with some variation depending on level of challenge and competence as defined by specific practice standards for facilitators. This paper will focus on the supervision training that was developed in July 2013 and the initial rollout of this across the country up to April 2014. The processes and elements of the training will be described and linked to the literature. The literature suggests supervision is dynamic, complex and requires training. Current challenges in multiple countries include an increased focus on business management within the helping professions. The various challenges and perceived benefits of the training will also be identified and discussed in relation to the literature, followed by identification of future focus areas and recommendations as a result of this review.

\section{Supervision training background}

The one-week training was developed by two contracted psychologists who had significant experience with the supervision and training of programme facilitators and the therapeutic and motivational programmes delivered. The author has approximately nine years' experience in the roles of programme facilitator, senior advisor of supervision implementation, supervisor, and line manager for programme facilitators. The seven initial trainers were invited to meet prior to running the courses to go through the training thoroughly with those who created it, in an attempt to promote consistency of approach and implementation. There were an initial five trainings co-facilitated around the country for eligible trainees between August and December 2013.

\section{Eligibility and assessment}

Eligibility criteria to be a trainee on the supervision training for supervisors included: (a) having achieved a standard of being fit to practice on two consecutive assessments (this represented between 3.5-4 years of functioning well in the role of programme facilitator); b) having no significant performance issues; c) regional and national approval; and d) that the trainee was interested in becoming a supervisor. This last point is underscored in the literature where being uninterested is identified as a barrier and a risk in relation to unwilling supervisors being more prone to take shortcuts and display behaviours implying a degree of neglect (Giddings, Cleveland, \& Smith, 2007).

Assessment of participants was designed to be cumulative throughout the week, starting on day two with skills practices. Trainees were assessed as "ready to supervise" (at beginner level), "further training/development needed" or "not recommended to supervise." The pathway and communication channels post-training for each of the three categories were clarified prior to training commencement. The two trainees assessed as needing further development completed follow-up plans in their regions and became "ready to supervise." One person was assessed as "not recommended to supervise" and the 48 assessed as "ready to supervise" 


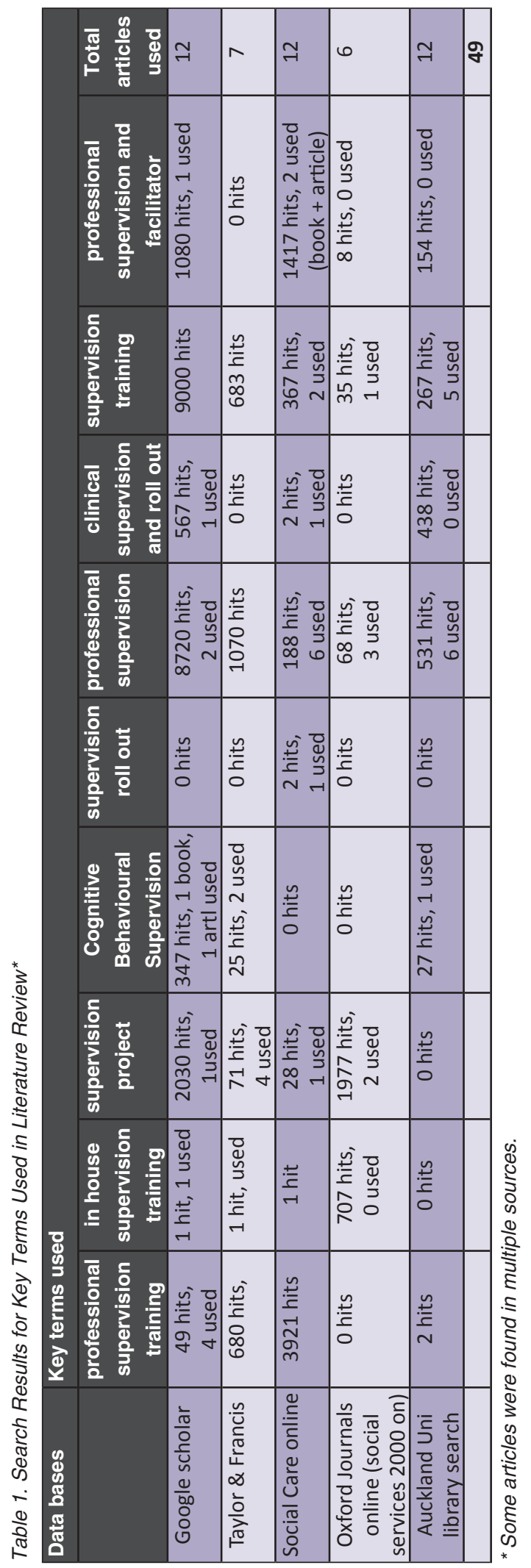

were approximately $30 \%$ of the estimated national programmes staff at the time. As the need for supervisors increased, a further four courses were delivered between July 2014 and November 2015, resulting in 33 more trained supervisors, a total of 78 (one person repeated the training after a significant time period).

\section{Search strategy and data}

In order to critically review this project and make recommendations for future training programme development, a literature review was retrospectively conducted. Literature specific to the corrections context or implementing supervision training was sparse (five articles from 32 located) so material from other helping professions was used and is referenced in this article. Key terms were used to search four databases and the library catalogue, as outlined in Table 1. Database searches focused on articles from 2000 to the present; however, this was not exclusive when articles or books were deemed particularly relevant. Where the number of hits for a term were $150+$ these were sorted by relevance, and the first 20-30 were considered.

Data from participant evaluation forms from eight of the nine, week-long supervision trainings were analysed, as well as results of 73 completed national supervision surveys done approximately six months after the initial implementation began.

\section{Findings}

Findings are presented from the participant evaluation data and feedback, and findings from the literature as to the benefits and barriers/challenges to supervision and supervision training, including specific challenges inherent in the DoC context. This evaluation was conducted as an internal process, not as a research study, and the presentation of data here is with the permission of the Department of Corrections. 


\section{Quantitative training evaluations}

Participants scored trainings on a scale of 1 (most positive) to 5 (least positive) which are displayed in Table 2. The mean and standard deviations for each aspect/statement were calculated over seven trainings. (Note: Evaluations from two training events were either unavailable or were not conducted.)

From these findings, it can be concluded that, overall, stronger aspects of the training from participants' perspectives were: the work done to introduce: practice with and build understanding of the model of supervision; the amount of skills practice and content throughout the training; the perceived effectiveness of the trainers; and the overall perceived usefulness of the training (shown by the readiness to recommend it to others). Areas for further focus within on-going support frameworks are working with ethical dilemmas involved in supervision and the building of feeling of being ready to supervise.

\section{Qualitative participant comments}

Within a space provided for written comments, main areas of positive feedback included: appreciation of the supportive and challenging environment; the "in the moment" regular feedback and coaching; the transparency of evaluation processes and the small group practices (as opposed to practising in front of the whole group); the benefit of line managers and facilitators being able to hear each other's anxieties when on the same training; and appreciation of discussion in the "frequently asked questions" section.

Suggestions for improvements to the training included: more modelling by trainers; more focus on ethical dilemmas; the inclusion of supervising some recorded practice; more focus on the writing of supervision reports and specific models; examples of not-so-good practice and reports; earlier discussion of trainee fears and blocks; pre-written role plays; more small group practice; the common themes in supervision and management of these (e.g., supervisee absenteeism); midway/midweek feedback from trainers; and follow-up training.

\section{Discussion}

\section{Benefits of supervision training and supervision}

In reviewing the findings of the evaluation of this training programme, it has been useful to go back to the literature to ascertain what has been written and researched about supervision training programmes. Multiple references were made in the literature to a

\section{Table 2. Training Ratings by Trainees}

\begin{tabular}{|l|l|l|}
\hline Statement \# & Aspects of the training & $\begin{array}{l}\text { Mean rating (1-5) and } \\
\text { (standard deviation) }\end{array}$ \\
\hline 1 & Trainees believe they are ready to supervise & $4.1(.63)$ \\
\hline 2 & The training content was sufficient & $4.5(.82)$ \\
\hline 3 & There was enough live practice in the training & $4.6(.80)$ \\
\hline 4 & The Seven Eyed Model will be useful & $4.8(.60)$ \\
\hline 5 & I understand the use of the CBT model in supervision & $4.5(.72)$ \\
\hline 6 & The trainers were effective & $4.8(.61)$ \\
\hline 7 & I would recommend the training to others & $4.9(.56)$ \\
\hline 8 & I believe I can act more ethically as a result & $4.3(.84)$ \\
\hline
\end{tabular}

Notes: -Two trainees numerical ratings were amended (qualitative comments were incongruent, possibly a misinterpretation of scores).

-Approx. $11 \%$ of feedback forms (8 of approx. 70) were absent. 
variety of benefits derived from supervision training over a range of occupations including counsellors, mental health nurses, social work field instructors, and in the field of treating trauma. To name a few, benefits included better outcomes from trained practitioners (Bambling, King, Raue, Schweitzer, \& Lambert, 2006); for staff, greater job satisfaction, increased knowledge and confidence, reduced feelings of burnout, improved professional solidarity, and other, more personal, development opportunities (Arvidsson, Löfgren, \& Fridlund, 2001; Burnard et al., 2003; Edwards et al., 2006; Rice et al., 2007). It is, however, difficult to comment precisely about what specific benefits resulted from the project, due to the fact that it was not initially set up as a research project, so it was without robust methods through which to evaluate the training and subsequent supervision. A national survey was conducted approximately six months after the initial training for new supervisors; however, there are a number of weak areas in relation to this process such as: the survey used was not specific to context, and supervisors and supervisee responses were not differentiated. In addition, variable implementation rates across regions meant that, for some areas, a percentage of supervisees would still be having supervision with existing supervisors (departmental psychologists) whereas other areas would have new supervisors in the role. The survey did not differentiate between these variables, and did not ask participants to identify and explore benefits but, rather, to rate aspects of supervision because the main focus was to find information about how supervision was faring in general across the country.

In spite of these weaknesses, 73 surveys were completed and, from a range of 26 questions, there is some initial insight. Overall the national average score of all questions was 4.22 out of a possible 5 (the scale being $5=$ best (almost always) and $1=$ worst (not at all) suggesting that satisfaction levels were generally high.
The five highest-rated responses were topics involving a supervisee being willing to learn; supervision being honest; involving a trusting relationship; being in a mutually agreed setting; and providing support. The range was between $4.63-4.5$ out of 5 . There is a positive theme of relationship here. The six lowest-rated responses were about having an experienced supervisor; making the most of opportunities; challenging and extending; enabling emotional expression; empowering and energizing; and professional development of the supervisee. The range was between $3.68-3.99$ out of 5, suggesting a lack of robust discussion within supervision.

\section{Barriers to supervision training and supervision}

Various barriers and challenges to supervision are highlighted in the literature, however, two main themes revolve around the inherent difficulty of empirically measuring effectiveness and results of supervision, and the interplay between supervision and the organisational culture in which supervision is operating (Davys \& Beddoe, 2010; O'Donoghue \& Tsui, 2013). In the DoC context, the additional challenge of client/offender characteristics is identified.

\section{The challenge of empirical research}

Due to the relatively recent and significant increase in supervision practice in human services, international and interprofessional consensus about central aspects of supervision is developing. For example, there is a theme in the literature confirming the need for supervision training and international consensus on this (Milne, Sheikh, Pattison, \& Wilkinson 2011; Gognsalvez \& Milne, 2010, as cited in Watkins \& Wang, 2014). However the complex, dynamic and subjective nature of supervision creates significant challenges in forming a strong empirical evidence base regarding training, the effectiveness of supervision, and outcomes-which is as yet not established. Although there are many examples of research, very few are 
methodically robust enough to provide conclusive and causal links around supervision and outcomes (Bennett \& Deal, 2012) and there is a lack of reliable, userfriendly tools to measure and research supervision and training (Younge \& Campbell, 2013, p. 1).

\section{The organisational cultures}

In the helping professions, supervision can be a place where practitioners interface with the organisational culture if supervision is provided internally. As identified by Middleman and Rhodes (1980) "the supervisor-worker relationship is the key encounter where the influence of organisational authority and professional identity collide, collude or connect" (p. 52, as cited in Webster, 2006, p. 226). The literature reports the prominence of supervision operating within organisational cultures, symptomatic of the "over-vigilant and bureaucratic" culture with a high focus on tasks, policies and rules, and a low focus on personal relatedness (Hawkins \& Shohet, 2012, p. 230). In this type of culture, resourcing can become dependent on efficiencies and performance measures (Egan, 2012) and the complex qualitative processes inherent to supervision can appear time-consuming and unproductive.

Although there were only three articles found specific to staff supervision (probation staff, not programme facilitators) in the DoC context, two recurring topics within the articles are the impact of an organisation's culture, and the impact of change management upon the work and staff, as seen in supervision. Although articles centred on introducing and engaging probation officers in supervision, there were no articles specific to supervision training. Webster (2006) explored the impact of transactional and transformational leadership styles on organisational culture with regard to DoC. Community Probation underwent significant changes between 1997 and 2002, resulting in a move away from supervision being informed by social work as a professional base, to being more aligned with practices within the private sector. Within supervision, this creates a range of experiences between engaging with a supervisor who has a transactional style, focused on tasks and policy; or a supervisor with a transformational style, focused on relationship and growth of the individual. This significantly impacts on supervision and, in this context, as Wood and Brown (2014) highlight, the supervisor influences the balance and tension between care and control for individuals, stating that "it is a constant challenge for the organisation to manage anxiety, responsibility and accountability, without becoming controlling, bureaucratic or autocratic" (2014, p. 333).

Specific to the corrections context, Wood and Brown give useful insight into some challenges of working with offenders, whom they describe as a "population who are often severely traumatised, with high levels of childhood adversity, who may have committed acts which have traumatised not just their victims, but also the perpetrators themselves" (2014, p. 330). Given the traits of offender populations and the inherently higher level of organisational attention to risk, the challenge can be further compounded by a risk-averse society. O’Donoghue, Baskerville, and Trlin (1998) explore supervision within the managerial climate of the DoC by analysing interview recordings of 15 probation staff. The inherent need for supervision within the context is highlighted, as well as the existing tension between the focus and value of organisational policies and practices, and the interests of the practitioners directly involved with clients and supervision. Supervision can be a place where the multitude of inherent risks and bonds created can be untangled and better understood to strengthen staff and inform practice.

The vulnerability created by the above challenges and barriers has been eloquently captured by $\mathrm{O}^{\prime}$ Donoghue et al., as they conclude how the foundations of professional 
supervision may be compromised when "laid in the shifting sand of expediency and efficiency rather than the sure ground of a service committed to best professional supervision practice" (1998, n.p.).

\section{Core themes from the literature review}

The training will now be discussed relative to the information and core themes found in the literature review. It will be divided in to two parts, the first being the content of the training, the second being the ways in which it was designed and delivered. Although most literature discussed is not empirical, and much research is deeply contextual, significant and useful themes emerge from the literature in regard to agreed ways to run supervision training, underlying principles and content topics. Seven topics for further development have then been identified and discussed regarding literature, and four on-going support structures for supervisors have been described.

\section{Content of supervision training}

There is consensus by multiple authors in the literature about the importance of topics to include in supervision training (Watkins \& Wang (2014, p. 182). Present in the literature and in the training (as can be seen in Figure 1), are the topics of ethics, supervision models, supervisor development, supervisor/supervisee roles and responsibilities, a model of therapist development, diversity, and research in regards to supervision (and programmes). Similarly, practice with agenda and goal setting, time management, assessment of supervisee needs, in situ feedback and prompting were present. Areas identified in the literature, but not included in training, were assessment/evaluation in supervision (developed post-training), establishment and maintenance of the supervision alliance, supervisory interventions/strategies, use of recorded practice and modelling by trainers. Whilst working with diversity was discussed, non-oppressive practice was not specifically explored.

\section{The design and delivery of supervision training}

There is general agreement in the literature about the way in which supervision training is accomplished: specifically, the importance of congruency between the methodology of the training course, the supervisory process, the aims of supervision, and the underlying principles. This is substantiated by discussion concerning the need for trainers to embody the core values of supervision in the processes of the training (Henderson, 2009, p. 3). Watkins and Wang have called these "supervision convictions", and advocate for these to be held sacrosanct by trainers and embodied throughout training (2014, p. 177).

The way the training was designed and delivered in the DoC project align well with these aspects of training design and delivery discussed in the literature. Aspects

\begin{tabular}{|l|}
\hline $\begin{array}{l}\text { A brief global history of programmes, criminology, } \\
\text { programme integrity and supervision }\end{array}$ \\
\hline Implementation FAQs \\
\hline The functions of supervision \\
\hline $\begin{array}{l}\text { The model of supervision - The Seven Eyed Model } \\
\text { (Hawkins \& Shohet, 2012) }\end{array}$ \\
\hline $\begin{array}{l}\text { The Tuakana-Teina concept and the Meihana model (Pitama, } \\
\text { Robertson, Cram, Gillies, Huria \& Dallas-Katoa, 2007) }\end{array}$ \\
\hline Power in supervision \\
\hline The supervision contract and forms. \\
\hline The CLEAR model (Hawkins \& Shohet, 2012) \\
\hline Ethical situations and boundaries \\
Common mistakes \\
\hline Ongoing support frameworks for supervisors. \\
\hline
\end{tabular}

Figure 1. Core Training Content 
of these will now be described more fully, starting with four areas of intentional parallel process between training and supervision sessions. There is a focus on the four most recent DoC training courses due to the author's higher involvement in co-delivering these.

1. Approach to training. Trainee supervisors were seen as "participants", their skills and experiences were valued as Casemore (2009) promotes, and they were actively encouraged to take responsibility for their learning. Trainers strove to embody the values and spirit of effective supervision by building participant self-efficacy, by giving and receiving feedback, by coaching, and by expressing clear belief in the capacity of participants. Alongside constructive feedback, this expression of belief seemed particularly important due to possible prior deferring to psychologist supervisors and connected feelings of self-doubt, as expressed by participants in some training courses. Exercises such as the competence matrix were used to support self-evaluation and development of the internal supervisor over the week.

2. Transparency and the setting of routines helped create a supportive, yet therapeutically demanding environment. For example: being transparent about the supportive role of the trainers; training evaluation processes; discussing expectations; ensuring regular space for reflections and concerns; daily opening and closing routines; and being responsive to participant needs.

3. Alongside direct teaching of theory, skills practice sessions with pairs or triads were started on day two and continued within each day so as to give participants multiple opportunities to practise skills and build their competence. Equally important, this gave trainers adequate opportunity to observe the participants' practice over the week, to discuss feedback and to "coax expertise" (Waskett, 2009, p. 230). Training by way of ensuring a mix of experiential learning, alongside theory within trainings is supported by the literature (Casemore, 2009; Hill, 2014, as cited by Milne et al., 2011, p. 63). Explicit timeframes were set for skills practices and focus points identified for feedback from peers.

4. As discussed by Bailey (2009), the design of a training programme and the manner of assessment are inseparable. As described above, skills practices were cumulative, building skills incrementally. As new models were learned, they could be put into practice; as skills and familiarity developed, realistic variables such as co-facilitator supervision were built in and more challenging issues brought to the practice. This had similar benefits to the collaborative and cumulative nature of a "Portfolio approach" (Bailey, 2009) as it allowed a more accurate assessment of a participant, and is congruent with the ongoing nature of the practice and spirit of supervision itself.

Bailey (2009) outlines other elements of training design and implementation that do not readily fit into the structure of a week of full-time training, but are important considerations for future up-skilling. This includes participants providing written feedback to fellow participants after skills practice sessions and written assignments. Elements of this could be implemented in skills development in writing evidence-based feedback in mock supervision reports.

Training was fairly streamlined due to the relative homogeneity of the work of participants and their potential supervisees. For example, all (bar one participant) were highly involved in and/or running, or had been running the DoC therapeutic programmes with offenders. Consequently, there is existing familiarity and use of similar models, theories and practices, as well as (for most) completion of the initial DoC programme facilitator training - all of which created a helpful common ground for supervision training. 


\section{Topics for further focus}

There are many important topics, and priorities will likely always need to be made due to time and resource constraints. From the literature, feedback, and overall evaluation of the initial week-long courses, seven topics are identified and outlined below which are important for follow-up within future training courses and within the support frameworks discussed further on.

1. Dual role relationships. As well as challenges, some studies do suggest benefits of internally provided supervision. Egan (2012), in a study of 675 social workers in managerial settings in Australia found that of the $66 \%$ workers who had supervision by their line manager, almost half said that internally provided supervision was the most useful; despite the fact that $50 \%$ of workers had had feedback linked to their performance appraisals. This suggests that the potential conflicts inherent in dual role relationships within internal supervision of staff by line managers need not be detrimental to supervision and can have their own strengths. However, multiple dynamic factors are involved which, inherently, create challenges and ethical dilemmas. Power dynamics, strategies, and helpful boundaries were discussed in training, however, throughout the working in the reality of these relationships, ongoing support is vital to maintain competence.

2. Ethics. Upholding ethical practice is essential to professional supervision and the dilemmas that arise can be complex and personal. As situations unfold in reality, theoretical understanding is often more challenging to put into practice. Consequently, although ethics was a topic included in the supervision training, it is an area best regularly discussed throughout the working reality of dilemmas that arise so that a grounded competence can be developed.
3. Anti-oppressive supervision. Jackson (1988) and Tsui and Ho (1997) outline findings in relation to gender and culture which suggest the "...monocultural Westminster system, within which the Community Probation Service operates, was the dominant organisational context and significantly influenced the participants' understanding of the processes used in professional supervision" (as cited in O'Donoghue et al., 1998, n.p.). This may or may not reflect the current reality several decades later; however, the high level of awareness required to practise in an anti-discriminatory and anti-oppressive manner makes this an important topic to regularly work with.

4. Using recorded practice. Using recordings of practice to specifically develop feedback skills, and to gain competence in evaluating future supervisees against measuring frameworks, can build the necessary familiarity and confidence to effectively carry out sensitive supervisory tasks. Likewise, gaining understanding and familiarity of the measurement tool through which supervisors may be assessed. The importance of the use of recorded practice is recognised in a study of supervision amongst Cognitive Behavioural Therapy (CBT) practitioners (Grant, 2009). Weak areas identified included the limited use of recorded practice (5\% used these regularly) or live observations ( $6 \%$ used these often).

5. Organisational challenges. Three aspects of the organisation feature in consideration of the challenges faced when implementing in-house supervision. These are organisational culture, the client profile and the location of the work in a public service environment.

\subsection{Organisational cultures. Within} the training there was space created to discuss aspects and impacts of 
the implementation of in-house supervision. As Clare (2001) identifies, one component of effective supervision is the ability to work through blocks, including potential challenges within the organisation's culture. For example, such challenges can include working through periods of heightened organisational change, or tension between target timeframes and the realities of reflective practice.

\subsection{Client characteristics and management.} Within the correctional context, the likelihood of increased potential for sub-conscious processes to be triggered and significantly influence the work (the working relationships and the workplace), is recognised by Wood and Brown (2014). By examining the dialogue of probation officers who had taken up the offer of individual supervision, specific areas were identified as being beneficial to discuss, such as how to work through the possible transference and counter-transference processes in play, and how to contain potential harm and still promote client responsibility (Wood \& Brown, 2014). Feelings of anxiety stemming from transference and counter-transference and vulnerability around being able to uphold appropriate authority throughout, were amongst those shared in supervision (Wood \& Brown, 2014). Logically, other staff working directly with clients in this context are likely to have similar areas to discuss.

5.3 Public service. Tension is also created when the organisation is a government department, visible and accountable to the public. Compounding this tension is the emotive aspect of offending, the layperson's understanding of risk, and the media, which all influence perceptions and judgement. Potential impacts to supervision can include a lesser focus on in-depth review of practice and an increased managerial focus (Beddoe, 2010; Davys \&
Beddoe, 2010), or a possible unhelpful dependency may be caused by the supervisee's apprehension regarding their role "...being less about the 'right decision' and more about a defensible decision" (Pollack, 2010, p. 1274).

6. Supervisees. Integral to effective supervision is a collaborative process which needs two informed and prepared parties rather than one. Morrison (2005) outlines many potential benefits to supervisees, supervisors and therefore to an organisation, of preparing supervisees. Within the project timeframe there were approximately six half-day supervisee courses run around the country. However, clarification of supervisee responsibilities and rights as well as other related topics are important to cover to some degree in initial supervision training, and to regularly re-visit in future supervisor courses, supervisee training and regular on-going support forums.

7. Supervisor development. Grant (2009) identifies two systems in the development of cognitive behavioural therapists, relevant to DoC facilitators of CBT-based programmes through the parallels between CBT and Cognitive Behavioural Supervision identified by Kavanagh, Bennett-Levy, and Crow (2002). There is the initial "declarative and procedural systems" which involve knowledge and facts and the novice supervisor mastering cognitive behavioural techniques. Secondly, there is the more advanced "reflective system" where techniques and skills are used sensitively according to the "moment by moment opportunities" involved (Kavanagh et al., 2002, p. 217). Inherent in developing this second system are practice and reflection. A model of supervisor development was discussed in training and is an important area to develop awareness and to actively work through the ongoing support structures. 


\section{On-going support for supervisors}

Research suggests that "...policy and practice statements are directed at how supervision itself can be delivered effectively, rather than addressing the question of how supervisors themselves can be supported and developed" (Milne, 2009 , p. 155). Creating a follow-up training attached to the initial training but some time later, is highly recommended. Within the project, various on-going support frameworks were created and implemented to support supervisory practice. These are outlined below and are also forums where the various topics mentioned earlier, can be further explored with the benefit of cumulative experience.

* Monthly Coaching Workshops (three hours each) for all practising supervisors within their region or area. These workshops aim to support ongoing supervisor development and to promote consistency and maintenance of effective supervision. Content naturally varies; however, regular viewing of recorded samples of facilitator (supervisee) practice is intended, to offer the opportunity to compare, discuss and develop consistent and effective supervisory and feedback skills.

* Weekly supervisors' supervision by a psychologist for approximately one hour per week. Within this, the viewing of some recorded practice (of the supervisee's supervisee) is important, to support accurate identification and prioritising of feedback and skill development. This sits alongside encouragement to occasionally bring recordings of supervision sessions to supervisors' supervision to use for learning.

* Supervision champions. This role requires a two- or three-hour commitment per month in each region or area. Tasks include gathering the area's main supervision challenges and achievements and discussing these via a nationally led audiovisual link once a month with other champions, and preparing monthly coaching workshops.

* Bi-annual audits and annual monitoring of supervision (developed post-training).
Basic audits support best practice in terms of keeping any paper work up to date and confidential. Annual monitoring of a random selection of supervision is a window into what happens in supervision and can be used to inform, support and celebrate.

Alongside recommending a second part to the initial training, other beneficial activities recommended are surveying and getting feedback from the regions about how the support structures are working so that any barriers or challenges can be worked through, running regular supervisee training, and creating a structure around the reviewing and updating of the supervision training.

\section{Conclusion}

There is significant and repeated recognition in the literature of the complexity and multifaceted nature of skills required of supervisors (Watkins \& Wang (2014) which has firmly placed supervisory practice as a profession in its own right. There are substantial developments which identify and validate the complexity of supervision and evidence the need for training. Watkins argues, as cited in Younge and Campbell (2013, p. 2), that " $[w]$ e would never dream of turning untrained therapists loose on needy patients, so why would we turn untrained supervisors loose on untrained therapists who help those needy patients?" (Watkins, 1997, p. 604).

Currently there is a plethora of supervision training offered and numerous benefits have been connected to supervision. Simultaneously there is recognition of the pressures currently placed on supervision connected to a "risk society" where organisations are focused on safety and responses to risk, and where policies encourage regulation, targeted intervention programmes and discourage autonomous decision making (Beddoe, 2010, p. 1280). This "growing dominance of business management approaches in human service organisations" (Hair, 2013, p. 1563) connects to a subsequent demand for supervision to be quantified and measurable. Challenges such as the above, as well those specific to the DoC context, such as 
offender characteristics and being in the public service, are significant but also highlight the need for supervision and supervision training.

When viewed through the lens of the literature, the DoC supervision training and implementation for programme facilitators reflects relatively well. Many of the training topics identified in the literature, and the ways in which the training was run, were present and congruent. Overall benefits of the project, as seen by the author, were an increased level of national consistency and familiarity in the approach to supervision, active awareness of the supervision model used, and a strengthened focus on programme integrity. Areas identified as being absent or in need of on-going attention have largely been considered as topics to be supported through the on-going support structures discussed and recommendations made.

However, whilst identification of these areas is initially helpful; following through on maintaining active support structures is another challenge given the multitude of competing organisational demands. This "embedded" nature of supervision means that, as Milne (2009) states, in supervision training it is important to continue to include ways to work through organisational factors that impinge on supervision. Likewise, increased attention to supervisee training is important, especially given that supervision strives to be a co-created trust-filled process; as well as more attention to the supervision of supervisors, this being classified by Milne (2009) as one of the most deficient areas within the profession.

Although the areas for further development within supervision are numerous, interconnected and require time, there is consistent acknowledgement in the literature that supervision is more than the sum of its parts. The creativity of informed yet spontaneous engagement in supervision which is responsive to the multiple nuances of the moment can result in a process where " $1+1=3$ " (Clare, 2001, p. 78) and latent potential is realised, which is a win-win for all involved.

\section{References}

Arvidsson, B., Löfgren, H., \& Fridlund, B. (2001). Psychiatric nurses' conceptions of how a group supervision programme in nursing care influences their professional competence: A 4-year follow-up study. Journal of Nursing Management, 9, 161-171.

Bailey, C. (2009). Recruitment and access. In P. Henderson (Ed.), Supervisor training issues and approaches (pp. 43-54). London, England: Karnac Books.

Bambling, M., King, R., Raue, P., Schweitzer, R., \& Lambert, W. (2006). Clinical supervision: Its influence on client rated working alliance and client symptom reduction in the brief treatment of major depression. Psychotherapy Research, 16, 317-331.

Beddoe, L. (2010). Surveillance or reflection: Professional supervision in "the risk society." British Journal of Social Work, 40, 1279-1296. doi:10.1093/bjsw/bcq018

Bennett, S., \& Deal, K. H. (2012). Supervision training: What we know and what we need to know. Smith College Studies in Social Work, 82, 2-3. doi:10.1080/00377317. 2012.693023

Burnard, P., Edwards, D., Hannigan, B., Fothergill, A., Coyle, D., Cooper, L., ... Adams, J. (2003). The effectiveness of clinical supervision on burnout amongst community mental health nurses in Wales. Journal of Psychiatric and Mental Health Nursing, 10, 231-238.

Casemore, R. (2009). It is all in the relationship: Exploring the differences between supervision training and counselling training. In P. Henderson (Ed.), Supervisor training issues and approaches (pp. 15-25). London, England: Karnac Books.

Clare, M. (2001). Operationalising professional supervision in this age of accountabilities. Australian Social Work, 54(2), 69-79. doi:10.1080/03124070108414325

Davys, A., \& Beddoe, L. (2010). Best practice in professional supervision: A guide for the helping professions. London, England: Jessica Kingsley.

Durie, M. (1998). Whaiora: Maoāri health development (2nd ed.). Auckland, NZ: Oxford University Press.

Edwards, D., Burnard, P., Hannigan, B., Cooper, L., Adams, J., Juggessur, T., ... Coyle, D. (2006). Clinical supervision and burnout: The influence of clinical supervision for community mental health nurses. Journal of Clinical Nursing, 15, 1007-1015. Egan, R. (2012). Australian social work supervision practice in 2007, Australian Social Work, 65(2), 171-184. doi:10.1080/031 2407X.2011.653575

Egan, R. (2012). Australian social work supervision practice in 2007, Australian Social Work, 65(2), 171-184, doi: 10.1080/0312407X.2011.653575

Giddings, M. M., Cleveland, P. H., \& Smith, C. H. (2007). Responding to inadequate supervision, The Clinical Supervisor, 25(1), 105-126. doi:10.1300/J001v25n01_08

Grant, A. (2009). Training for supervising cognitivebehavioural practitioners and others. In P. Henderson (Ed.). Supervisor training issues and approaches. (pp. 209-222). London, England: Karnac Books.

Hair, H. J. (2013). The purpose and duration of supervision, and the training and discipline of supervisors: What social workers say they need to provide effective services. British Journal of Social Work, 43(8), 1562-1588. doi:10.1093/bjsw/bcs071 
Hawkins, P., \& Shohet, R. (2012). Supervision in the helping professions ( $4^{\text {th }}$ ed. $)$. Maidenhead, UK: Open University Press

Henderson, P. (2009). Supervisor training issues and approaches. London, UK: Karnac Books Ltd.

Hill, C. E. (2014). Helping skills training. In C. E. Watkins \& D. L. Milne, (Eds.), Wiley international handbook of clinical supervision (pp. 329-341). Chichester, NY: John Wiley \& Sons. doi:10.1002/9781118846360

Kavanagh, D. J., Bennett-Levy, J., \& Crow, N. (2002). A cognitive behavioural approach to supervision. In M. McMahon \& W. Patton (Eds.), Supervision in the helping professions a practical approach (pp. 131-141). Frenchs Forest, NSW: Prentice Hall.

Milne, D. (2009). Evidence-based clinical supervision. Principles and practice. Chichester, England: John Wiley \& Sons.

Milne, D. L., Sheikh, A. I., Pattison, S., \& Wilkinson, A. (2011). Evidence-based training for clinical supervisors: A systematic review of 11 controlled studies, The Clinical Supervisor, 30(1), 53-71. doi:10.1080/07325223.2011. 564955

Morrison, T. (2005). Strength to strength. A facilitator's guide to preparing supervisees, students and trainees for supervision. Brighton, England: Pavilion Publishing.

O'Donoghue, K. B., Baskerville, M. A., \& Trlin, A. D. (1988). Professional supervision in the new managerial climate of the Department of Corrections. New Zealand Association of Social Workers Inc. Aotearoa Conference, Hamilton, New Zealand.

O'Donoghue, K., \& Tsui, M. (2013). Social work supervision research (1970-2010): The way we were and the way ahead. British Journal of Social Work, 45, 616-633. doi:10.1093/bjsw/bct115

Pitama, S., Robertson, P., Cram, F., Gillies, M., Huria, T., \& Dallas-Katoa, W. (2007). Meihana model: A clinical assessment framework. New Zealand Journal of Psychology, 36(3), 118-125.

Pollack, S. (2010). Labelling clients 'risky': Social work and the neo-liberal welfare state. British Journal of Social Work, 40(4), 1263-1278. doi:10.1093/bjsw/bcn079

Rice, F., Cullen, P., McKenna, H., Kelly, B., Keeney, S., \& Richey, R. (2007). Clinical supervision for mental health nurses in Northern Ireland: Formulating best practice guidelines. Journal of Psychiatric and Mental Health Nursing, 14, 516-521. Waskett, C. (2009). Learning to supervise using a solution-focused approach. In P. Henderson (Ed.), Supervisor training issues and approaches (pp. 223-230). London, England: Karnac Books.

Watkins, C.E. (1997). Some concluding thoughts about psychotherapy supervision, in Handbook of psychotherapy supervision, Watkins C.E. (Ed.) (pp.603-616) New York NY: Wiley

Watkins, C. E., \& Wang, C. D. C. (2014). On the education of clinical supervisors. In C. Watkins \& D. Milne (Eds.) Wiley international handbook of clinical supervision (pp. 177-203). Chichester, NY: John Wiley \& Sons.

Webster, M. (2006). Professional supervision as continuing professional education: Recent Community Probation Service experience in a turbulent environment. In L. Beddoe \& J. G. Jesson (Eds.), Continuing professional education: Out of bounds and borders:
Proceedings of the conference (pp. 224-231). Auckland, NZ: University of Auckland.

Wood, H., \& Brown, G. (2014). Psychoanalytically-informed clinical supervision of staff in probation services. Psychoanalytic Psychotherapy, 28(3), 330-344. doi:10.1080/02668734.2014.922248

Younge, J., \& Campbell, A. (2013). A qualitative study exploring the perceived impact of supervision training on cognitive therapy supervisor practice. The Cognitive Behaviour Therapist, 6(e1), 1-14. doi:10.1017/ S1754470X13000068 